ANNOTATION

\title{
CAUSES OF DEATH OF EX-BOXERS
}

\section{J. L. BLONSTEIN - London and L. SCHMID, M.U.Dr., C.Sc. - Prague}

Whenever an outstanding athlete dies, the question arises whether competitive athletics is noxious. The team managers and coaches, as a rule, declare in unison that physical training does enormous good and prolongs human life. A good many people do acknowledge the usefulness of adequate and regular physical training, but consider competitive athletics a harmful and life-shortening factor. Any comparison with men from the remaining population will be necessarily very difficult. Athletes form a certain selected group because only a healthy individual, as a rule, indulges in physical training.

An attainment of longevity is a product of a great variety of factors. Heredity plays a prime role. Further, it has to be considered that the human organism is a compact whole, in which the nervous component plays a decisive role. Longevity can be expected with an individual whose functional structure is associated with a nervous system capable of excitation. Any factor maintaining lively nervous activity prolongs the life span. All people who achieved high ages had always been hard-working and physically active.

Ageing is a biological process, the first signs of which are very difficult to apprehend. Such changes arise at different periods in different individuals. The early manifestations of ageing likewise vary considerably. The initial stage of ageing is always a product of processes which are reversible to a considerable extent and whose advance can be retarded or even annulled by exercise, gymnastics, athletics, and games.

Consequently, prolongation of the life span is a result of a variety of factors. It is also a social problem. Thanks to improving hygienic conditions, decline in inifant mortality, and systematic fight against infections, human life has been gradually prolonged. Ample evidence is available at present that motion safeguards longevity. Systematic and regular exercise is reflected in many favourable indicators in an individual. It is an established fact that physical training, indulged in throughout the lifetime, delays the physiological decline of efficiency in old age and reduces the biological age.

Several earlier papers of ours had been dedicated to this theme. At the very beginning of our study we realized that it is practically impossible to single out the effect of athletic activity alone on human longevity. Human life is primarily formed and governed by the social system. Past war-time sufferings, the precipitate life pace, and a great variety of other factors and circumstances participate in wearing out the organism. Long ago, Academician Bogomolets has written that the art of prolonging human life must begin from stopping to shorten it!

Our studies, conducted over many years, have shown that with the exception of individuals who had died in consequence of external violence, the mean age of competitive athletes was higher than that of the remaining male population. Besides, we have confirmed the well-known date concerning the favourable effect of matrimony on the husband's life span.

On this occasion, we would like to discuss briefly the causes of death of ex-boxers. We succeeded in collecting pertinent data concerning 59 ex-boxers. Their number is too small to allow any definite conclusions being drawn; nonetheless, some data are interesting.

As in our earlier papers, and the present one, we have to consider cases of death caused by external force.

An athlete, as a rule, relies upon his perfect health, strength, and performance, and in consequence often runs unnecessary risks. This may be the reason why Dublin says in one of his studies that he is not endowed for a long life. We know that weak and physically less efficient individuals may sometimes survive much more robust ones. The former, of course, take better care of themselves, and run no risks.

Besides, we must not forget that athletes, as a rule, travel fairly often, and therefore are exposed to a greater hazard of traffic accidents. On the other hand, one must not disregard the fact that competition in certain branches of athletics likewise involves certain hazards for the participants. Boxing, no doubt, is such a branch.

Out of the 59 ex-boxers constituting our group of subjects, almost $74.5 \%$ died in consequence of external violence. It is a high percentage, but those boxers are also included who had died in the course of the Second World War.

The deaths due to external violence in the course of this war were road accidents, accidents at work, suicide and some sub-dural haematomata due to some injuries in the ring. 
We do not know the condition of the coronary arteries and the brain blood vessels.

The causes of natural death were:

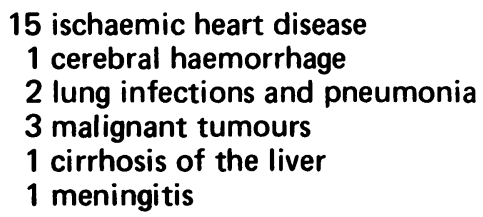

The average age of those dying from natural causes was 62.
The small number of ex-boxers who had died a natural death (15 altogether) does not allow us to draw any meaningful conclusions in the present preliminary communication. More data are needed. We expect to supply ampler information about this topic in the future. The present preliminary analysis of ours only documents, for the time being, that in life span, ex-boxers did not essentially differ from the remaining male population. No difference has been found in the causes of death, either.

Roberts reporting on 243 ex-professional boxers found evidence of "brain damage" in $17 \%$. The findings and neurological lesions were no different on percentage from men who had not boxed, and from women.

\section{ANNOTATION}

INJURIES SUSTAINED AT THE 1ST WORLD AMATEUR CHAMPIONSHIPS, HAVANA, CUBA

August 17-30, 1974

$$
258 \text { Boxers - } 252 \text { Bouts }
$$

There were $19 \mathrm{~K}$ 'Os. Only three were unconscious: two minutes, one minute and three minutes. The latter was removed to hospital. Detained overnight; no complications. E.E.G. normal.

There were 14 bouts stopped with cuts around the eyes (6 sutured).

There were 13 small cuts (bouts not stopped). There were 5 haematomata - not stopped. There was one fractured? nose; no complications. There were two abrasions; not stopped. One fractured clavicle. Two fractured metacarpals.

J. L: BLONSTEIN, O.B.E.

President - Medical Commission, A.I.B.A. 